\title{
Existence of a solution for a three-point boundary value problem for a second-order differential equation at resonance
}

Juan J Nieto*

${ }^{\text {*Correspondence: }}$

juanjose.nieto.roig@usc.es Departamento de Análisis

Matemático, Facultad de

Matemáticas, Universidad de

Santiago de Compostela, Santiago

de Compostela, 15782, Spain

Department of Mathematics,

Faculty of Science, King Abdulaziz

University, P.O. Box 80203, Jeddah,

21589, Saudi Arabia

\begin{abstract}
We present a new existence result for a second-order nonlinear ordinary differential equation with a three-point boundary value problem when the linear part is noninvertible.

MSC: Primary 34B10; secondary 34B15

Keywords: nonlinear ordinary differential equation; three-point boundary value problem; problem at resonance; existence of solution
\end{abstract}

\section{Introduction}

The study of multi-point boundary value problems for linear second-order ordinary differential equations goes back to the method of separation of variables [1]. Also, some questions in the theory of elastic stability are related to multi-point problems [2]. In 1987, Il'in and Moiseev [3, 4] studied some nonlocal boundary value problems. Then, for example, Gupta [5] considered a three-point nonlinear boundary value problem. For some recent works on nonlocal boundary value problems, we refer, for example, to [6-15] and references therein.

As indicated in [16], there has been enormous interest in nonlinear perturbations of linear equations at resonance since the seminal paper of Landesman and Lazer [17]; see [18] for further details.

Here we study the following nonlinear ordinary differential equation of second order subject to the three-point boundary condition:

$$
\begin{aligned}
& -u^{\prime \prime}(t)=f(t, u(t)), \quad t \in[0, T], \\
& u(0)=0, \quad \alpha u(\eta)=u(T),
\end{aligned}
$$

where $T>0, f:[0, T] \times \mathbb{R} \rightarrow \mathbb{R}$ is a continuous function $\alpha \in \mathbb{R}$ and $\eta \in(0, T)$.

In this paper we consider the resonance case $\alpha \eta=T$ to obtain a new existence result. Although this situation has already been considered in the literature [19], we point out that our approach and methodology is different.

(c) 2013 Nieto; licensee Springer. This is an Open Access article distributed under the terms of the Creative Commons Attribution License (http://creativecommons.org/licenses/by/2.0), which permits unrestricted use, distribution, and reproduction in any medium, provided the original work is properly cited. 


\section{Linear problem}

Consider the linear second-order three-point boundary value problem

$$
\begin{aligned}
& -u^{\prime \prime}(t)=\sigma(t), \quad t \in[0, T], \\
& u(0)=0, \quad \alpha u(\eta)=u(T)
\end{aligned}
$$

for a given function $\sigma \in C[0, T]$.

The general solution is

$$
u(t)=c_{1}+c_{2} t-\int_{0}^{t}(t-s) \sigma(s) d s
$$

with $c_{1}, c_{2}$ arbitrary constants.

From $u(0)=0$, we get $c_{1}=0$. From the second boundary condition, we have

$$
(T-\alpha \eta) c_{2}=\int_{0}^{T}(T-s) \sigma(s) d s-\alpha \int_{0}^{\eta}(\eta-s) \sigma(s) d s .
$$

\subsection{Nonresonance case}

If $\alpha \eta \neq T$, then

$$
c_{2}=\frac{1}{T-\alpha \eta}\left[\int_{0}^{T}(T-s) \sigma(s) d s-\alpha \int_{0}^{\eta}(\eta-s) \sigma(s) d s\right]
$$

and the linear problem (2) has a unique solution for any $\sigma \in C[0, T]$. In this case, we say that (2) is a nonresonant problem since the homogeneous problem has only the trivial solution as a solution, i.e., when $\sigma=0, c_{1}=c_{2}=0$ and $u=0$. Note that the solution is given by

$$
u(t)=\int_{0}^{T} g(t, s) \sigma(s) d s
$$

with

$$
g(t, s)= \begin{cases}\frac{t(T-s)}{T-\alpha \eta}-\frac{t \alpha(\eta-s)}{T-\alpha \eta}-(t-s), & 0 \leq s<\min (\eta, t), \\ \frac{t(T-s)}{T-\alpha \eta}-\frac{t \alpha(\eta-s)}{T-\alpha \eta}, & 0 \leq t<s<\eta<T, \\ \frac{t(T-s)}{T-\alpha \eta}-(t-s), & 0 \leq \eta<s<t \leq T, \\ \frac{t(T-s)}{T-\alpha \eta}, & \max (\eta, t)<s \leq T .\end{cases}
$$

For $T=1$ this is precisely the function given in Lemma 2.3 of [20] or in Remark 12 of [21].

\subsection{Resonance case}

If $T=\alpha \eta$, then (3) is solvable if and only if

$$
\int_{0}^{T}(T-s) \sigma(s) d s=\alpha \int_{0}^{\eta}(\eta-s) \sigma(s) d s
$$


and then (2) has a solution if and only if (5) holds. In such a case, (2) has an infinite number of solutions given by

$$
u(t)=c t-\int_{0}^{t}(t-s) \sigma(s) d s, \quad c \in \mathbb{R} .
$$

In particular $c t, c \in \mathbb{R}$ is a solution of the homogeneous linear equation

$$
-u^{\prime \prime}(t)=0, \quad t \in[0, T]
$$

satisfying the boundary conditions

$$
u(0)=0, \quad \alpha u(\eta)=u(T) .
$$

Note that

$$
u(T)-u(\eta)=c_{2} T-\int_{0}^{T}(T-s) \sigma(s) d s-c_{2} \eta+\int_{0}^{\eta}(\eta-s) \sigma(s) d s,
$$

and then

$$
c_{2}=\frac{1}{T-\eta}\left[u(T)-u(\eta)+\int_{0}^{T}(T-s) \sigma(s) d s-\int_{0}^{\eta}(\eta-s) \sigma(s) d s\right] .
$$

We now use that $u(T)=\frac{T}{\eta} u(\eta)$ to get

$$
\frac{1}{T-\eta}[u(T)-u(\eta)]=\frac{1}{T} u(T)
$$

and

$$
c_{2}=\frac{1}{T-\eta}\left[\int_{0}^{T}(T-s) \sigma(s) d s-\int_{0}^{\eta}(\eta-s) \sigma(s) d s\right]+\frac{1}{T} u(T) .
$$

Hence the solution of (2) is given, implicitly, as

$$
u(t)=\int_{0}^{T} \frac{t(T-s)}{T-\eta} \sigma(s) d s-\int_{0}^{\eta} \frac{t(\eta-s)}{T-\eta} \sigma(s) d s-\int_{0}^{t}(t-s) \sigma(s) d s+\frac{t}{T} u(T)
$$

or, equivalently,

$$
u(t)=\int_{0}^{T} k(t, s) \sigma(s) d s+\frac{t}{T} u(T)
$$

where

$$
k(t, s)= \begin{cases}s, & 0 \leq s<\min (\eta, t), \\ t, & 0 \leq t<s<\eta \leq T, \\ \frac{t(T-s)}{T-\eta}-(t-s), & 0 \leq \eta<s<t \leq T, \\ \frac{t(T-s)}{T-\eta}, & \max (\eta, t)<s \leq T .\end{cases}
$$

We note that $k \in C([0, T] \times[0, T], \mathbb{R})$ and $k(t, s) \geq 0$ for every $(t, s) \in[0, T] \times[0, T]$. 


\section{Nonlinear problem}

Defining the operators:

$$
\begin{aligned}
& F: C[0, T] \rightarrow C[0, T], \\
& {[F u](t)=f(t, u(t)), \quad u \in C[0, T], t \in[0, T],} \\
& K: C[0, T] \rightarrow C[0, T], \\
& {[K \sigma](t)=\int_{0}^{T} k(t, s) \sigma(s) d s, \quad \sigma \in C[0, T], t \in[0, T],} \\
& L: C[0, T] \rightarrow C[0, T], \\
& {[L u](t)=\frac{t}{T} u(T), \quad u \in C[0, T], t \in[0, T],}
\end{aligned}
$$

the nonlinear problem is equivalent to

$$
u=N u,
$$

where $N=K \circ F+L$.

We note that (6) can be written as

$$
u(t)-\frac{t}{T} u(T)=\int_{0}^{T} k(t, s) \sigma(s) d s
$$

and the nonlinear problem (1) as

$$
u(t)-\frac{t}{T} u(T)=\int_{0}^{T} k(t, s) f(s, u(s)) d s .
$$

This suggests to introduce the new function $v(t)=u(t)-\frac{t}{T} u(T)$. To find a solution $u$, we have to find $v$ and $u(T)$.

For every constant $c \in \mathbb{R}$, we solve

$$
v(t)=\int_{0}^{T} k(t, s) f\left(s, v(s)+\frac{s}{T} c\right) d s
$$

and let $\varphi(c)$ be the set of solutions of (7). This set may be empty (no solution), a singleton (unique solution) or with more than one element (multiple solutions). For every $v_{c} \in \varphi(c)$, we consider

$$
u_{c}(t)=v_{c}(t)+\frac{t}{T} c
$$

and hence

$$
u_{c}(t)=\int_{0}^{T} k(t, s) f\left(s, u_{c}(s)\right) d s+\frac{t}{T} c .
$$

If $c=u_{c}(T)$, then $u_{c}$ is a solution of the nonlinear problem (1). We then look for fixed points of the map

$$
c \in \mathbb{R} \longrightarrow u_{c}(T) \in \mathbb{R}
$$


For $c \in \mathbb{R}$ fixed, we try to solve the integral equation (7).

Assume that there exist $a, b \in C[0, T]$ and $\alpha \in[0,1)$ such that

$$
|f(t, u)| \leq a(t)+b(t)|u|^{\alpha}
$$

for every $t \in[0, T], u \in \mathbb{R}$.

For $v \in C[0, T]$, define $F_{c} v \in C[0, T]$ as

$$
\left[F_{c} v\right](t)=f\left(t, v(t)+\frac{t}{T} c\right)
$$

Thus, a solution of (7) is precisely a fixed point of $K \circ F_{c}=K_{c}$. Note that $K_{c}$ is a compact operator. For $v \in C[0, T]$, let $\|v\|=\sup _{t \in[0, T]}|v(t)|$.

For $\lambda \in(0,1)$, if $v=\lambda K_{c}(v)$ we have

$$
v(t)=\lambda \int_{0}^{T} k(t, s) f\left(s, v(s)+\frac{s}{T} c\right) d s
$$

and

$$
|v(t)| \leq\|k\| \int_{0}^{T} f\left(s, v(s)+\frac{s}{T} c\right) d s \leq\|k\| \cdot T\left[\|a\|+\|b\|(\|v\|+c)^{\alpha}\right] .
$$

Hence there exist constants $a_{0}, b_{0}$ such that

$$
\|v\| \leq a_{0}+b_{0}(\|v\|+c)^{\alpha}
$$

for any $v \in C[0, T]$ and $\lambda \in(0,1)$ solution of $v=\lambda K_{c}(v)$. This implies that $v$ is bounded independently of $\lambda \in(0,1)$, and hence by Schaefer's fixed point theorem (Theorem 4.3.2 of [22]), $K_{c}$ has at least a fixed point, i.e., for given $c$, equation (7) is solvable.

Now suppose $f$ is Lipschitz continuous.

Then there exists $l>0$ such that

$$
|f(t, x)-f(t, y)| \leq l|x-y|
$$

for every $t \in[0, T]$ and $x, y \in \mathbb{R}$.

Then, for $v, w \in C[0, T]$, we have

$$
\left|\left[K_{c} v\right](t)-\left[K_{c} w\right](t)\right| \leq \int_{0}^{T} k(t, s) l|v(s)-w(s)| d s
$$

and

$$
\left\|K_{c} v-K_{c} w\right\| \leq\|k\| \cdot l \cdot T\|v-w\| .
$$

Thus, for $l>0$ small, equation (7) has a unique solution in view of the classical Banach contraction fixed point theorem. 
Now, under conditions (8) and (10), set

$$
c \in \mathbb{R} \longrightarrow v_{c} \in C[0, T]
$$

where $v_{c}$ is the unique solution of (7), and as a consequence of the contraction principle, this map is continuous.

Define the map

$$
\begin{aligned}
& \varphi: \mathbb{R} \longrightarrow \mathbb{R}, \\
& \varphi(c)=v_{c}(T) .
\end{aligned}
$$

If there exists $c \in \mathbb{R}$ such that $\varphi(c)=0$, then for that $c$ we have $v_{c}(T)$, and the function

$$
u_{c}(t)=v_{c}(t)+\frac{t}{T} c
$$

is such that $u_{c}(T)=c$, and therefore $u_{c}$ is a solution of the original nonlinear problem (1).

Now, assume that

$$
\lim _{u \rightarrow \pm \infty} f(t, u)= \pm \infty
$$

uniformly on $t \in[0, T]$.

Then the growth of $\|v\|$ is sublinear in view of estimate (9). However, $c$ growths linearly. Hence the norm of the function

$$
v_{c}(s)+\frac{s}{T} c
$$

growths asymptotically as $c$.

This implies that $\lim _{c \rightarrow \pm \infty} \varphi(c)= \pm \infty$, and there exists $c \in \mathbb{R}$ with $\varphi(c)=0$.

We have the following result.

Theorem 3.1 Suppose that $f$ satisfies the growth conditions (8) and (10). If (11) holds, then (1) is solvable for l sufficiently small.

Note that condition (11) is crucial since for $f(t, u)=\sigma(t)$ and, in view of (5), the problem (1) may have no solution.

\section{Competing interests}

The author declares that he has no competing interests.

\section{Acknowledgements}

This research has been partially supported by Ministerio de Economía y Competitividad (Spain), project MTM2010-15314, and co-financed by the European Community fund FEDER. The author is thankful to the referees for their useful suggestions. 


\section{References}

1. Gregus, M, Neumann, F, Arscott, FM: Three-point boundary value problems for differential equations. J. Lond. Math. Soc. 3, 429-436 (1971)

2. Timoshenko, S: Theory of Elastic Stability. McGraw-Hill, New York (1961)

3. Il'in, VA, Moiseev, El: Nonlocal boundary value problem of the first kind for a Sturm-Liouville operator in its differential and finite difference aspects. Differ. Equ. 23, 803-810 (1987)

4. Il'in, VA, Moiseev, El: Nonlocal boundary value problem of the second kind for a Sturm-Liouville operator. Differ. Equ. 23, 979-987 (1987)

5. Gupta, CP: Solvability of a three-point nonlinear boundary value problem for a second order ordinary differential equation. J. Math. Anal. Appl. 1683, 540-551 (1992)

6. An, Y: Existence of solutions for a three-point boundary value problem at resonance. Nonlinear Anal. 65, 1633-1643 (2006)

7. Franco, D, Infante, G, Zima, M: Second order nonlocal boundary value problems at resonance. Math. Nachr. 284, $875-884(2011)$

8. Han, X, Gao, H, Xu, J: Existence of positive solutions for nonlocal fourth-order boundary value problem with variable parameter. Fixed Point Theory Appl. 2011, Article ID 604046 (2011)

9. Infante, G, Zima, M: Positive solutions of multi-point boundary value problems at resonance. Nonlinear Anal. 69 2458-2465 (2008)

10. Pietramala, P: A note on a beam equation with nonlinear boundary conditions. Bound. Value Probl. 2011, Article ID $376782(2011)$

11. Ma, R: A survey on nonlocal boundary value problems. Appl. Math. E-Notes 7, 257-279 (2007)

12. Webb, JRL, Infante, G: Positive solutions of nonlocal boundary value problems: a unified approach. J. Lond. Math. Soc. 74, 673-693 (2006)

13. Webb, JRL, Zima, M: Multiple positive solutions of resonant and non-resonant nonlocal boundary value problems. Nonlinear Anal. 71, 1369-1378 (2009)

14. Zhang, P: Existence of positive solutions for nonlocal second-order boundary value problem with variable parameter in Banach spaces. Fixed Point Theory Appl. 2011, Article ID 43 (2011)

15. Zhang, H-E, Sun, J-P: Positive solutions of third-order nonlocal boundary value problems at resonance. Bound. Value Probl. 2012, Article ID 102 (2012)

16. Korman, P: Nonlinear perturbations of linear elliptic systems at resonance. Proc. Am. Math. Soc. 140, $2447-2451$ (2012)

17. Landesman, EM, Lazer, AC: Nonlinear perturbations of linear elliptic boundary value problems at resonance. J. Math. Mech. 19, 609-623 (1970)

18. Ambrosetti, A, Prodi, G: A Primer of Nonlinear Analysis. Cambridge University Press, Cambridge (1993)

19. Ma, R: Multiplicity results for a three-point boundary value at resonance. Nonlinear Anal. 53, 777-789 (2003)

20. Lin, B, Lin, L, Wu, Y: Positive solutions for singular second order three-point boundary value problems. Nonlinear Anal. 66, 2756-2766 (2007)

21. Webb, JRL: Positive solutions of some three-point boundary value problems via fixed point index theory. Nonlinear Anal. 47, 4319-4332 (2001)

22. Smart, DR: Fixed Point Theorems. Cambridge University Press, Cambridge (1974)

doi:10.1186/1687-2770-2013-130

Cite this article as: Nieto: Existence of a solution for a three-point boundary value problem for a second-orde

differential equation at resonance. Boundary Value Problems 2013 2013:130.

\section{Submit your manuscript to a SpringerOpen ${ }^{\circledR}$ journal and benefit from:}

- Convenient online submission

- Rigorous peer review

Immediate publication on acceptance

- Open access: articles freely available online

- High visibility within the field

- Retaining the copyright to your article 